\title{
BRIEF REPORT \\ Screening for Student Subjective Wellbeing: \\ An Analog Evaluation of Broad and Targeted Models
}

Tyler L. Renshaw ${ }^{1}$ and Jeffrey S. Chenier ${ }^{2}$

${ }^{1}$ Utah State University, Department of Psychology, Logan, UT.

${ }^{2}$ Lusher Charter School, New Orleans, LA.

Author note: We declare no financial conflicts of interests in conducting or reporting this work. Correspondence should be addressed to Tyler Renshaw at tyler.renshaw@usu.edu.

Version note: Final version of paper accepted for publication in Journal of Psychoeducational Assessment on 07-31-18. This version is not the copy of record and may not exactly replicate the final, authoritative version of the article. Upon publication, the final article will be available via https://doi.org/10.1177/0734282916678494 


\begin{abstract}
This brief report presents a secondary analysis of responses to the Student Subjective Wellbeing Questionnaire (SSWQ) with a sample of urban middle-schoolers. Relative classification validity evidence was evaluated for two screening models derived from responses to the SSWQ: one based on the Overall Wellbeing Scale (OWS) and the other based solely on the Academic Efficacy Subscale (AES). Results from Bayesian t-tests, using several school-reported outcomes as dependent variables, indicated evidence in favor of classification validity for both the OWS and AES screening models. Yet findings also show that the evidence for the AES model was stronger than that for the OWS model. Implications for future research and practice are discussed.
\end{abstract}

Keywords: screening; school mental health; student subjective wellbeing; assessment 


\section{BRIEF REPORT}

\section{Screening for Student Subjective Wellbeing:}

\section{An Analog Evaluation of Broad and Targeted Models}

Universal mental health screening is increasingly recognized as a pivotal practice for informing school-based prevention efforts (Dowdy, Ritchey, \& Kamphaus, 2010). Yet there is little agreement regarding how such screening should be conducted to optimize student outcomes, especially in secondary settings. One of the major parameters along which screening approaches vary is the behavioral domains or constructs targeted by a given instrument. The most common approach to mental health screening targets psychopathology or problem behavior (e.g., Eklund \& Dowdy, 2014), yet alternative approaches include targeting both problems and wellbeing in tandem (e.g., Kim, Furlong, Dowdy, \& Felix, 2014) or targeting wellbeing instead of problems (e.g., Renshaw, Long, \& Cook, 2015). To date, the latter approach - screening for student wellbeing alone-is arguably the least developed within the school mental health screening literature. Although there may be several reasons for this, one obvious influence is the factor of historical time. Whereas screening for student problems has been a viable endeavor since at least the 1970s (e.g., Cowen et al., 1973), the possibility of screening for student wellbeing emerged only within the past dozen years, as the positive psychology movement (Seligman \& Csikszentmihalyi, 2001) made its way into schools (Furlong, Gilman, \& Huebner, 2014).

The construct of wellbeing is used across the social and behavioral sciences to refer to all manner of healthy and successful human functioning. To date, mental health screening in schools has largely focused on subjective wellbeing, which can be operationalized as 
self-appraisals of one's private behavior (thoughts and feelings) and public behavior (verbal and physical actions) that are deemed personally or socially desirable (Renshaw, 2016). The evidence in favor of screening for subjective wellbeing is primarily derived from findings showing meaningful relationships between measures of general or overall wellbeing and a variety of concurrent valued life outcomes (see Arslan \& Renshaw, 2018, for a brief review). Although promising, there are at least two limitations of this emerging evidence base. First, the analytic approaches have mostly made use of continuous variables, suggesting questionable generalizability to the actual practice of screening, which converts responses from continuous measures into categorical classification variables. Second, the analytic approaches have rarely compared the relative validity of broad versus targeted screening models, simply assuming that screening for general or overall wellbeing is likely to be more useful than screening for more particular wellbeing constructs. The rationale for taking a broad approach has been grounded in analogy to the intelligence assessment literature, where the construct of $g$-representing general or overall intellectual ability-is considered a better predictor of student performance than any particular, isolated ability (Furlong, You, Renshaw, Smith, \& O'Malley, 2014). Yet this rationale seems questionable in light of best-practice screening recommendations, which suggest that constructs tapped by screeners be squarely aligned with the criterion(s) of interest, and no more (Glover \& Albers, 2007). It therefore seems reasonable to hypothesize that targeted screening models may actually have greater predictive power than broad models, as construct-criterion alignment is better specified and more closely matched in the former compared to the latter. 
To our knowledge, only one study has been conducted so far to probe the relative validity of broad versus targeted wellbeing screening models. Specifically, Renshaw and Chenier (2016) investigated the relationship between responses to the Student Subjective Wellbeing Questionnaire (SSWQ; Renshaw et al., 2015) and several school-reported outcomes, including standardized achievement test scores, grade-point average, and an indicator of general behavioral performance. Their analytic approach tested these relationships at both the broad level, represented by the SSWQ's overall wellbeing scale/factor, and the targeted level, represented by the measure's subscales/factors: joy of learning, educational purpose, school connectedness, and academic efficacy. Results showed that although the overall wellbeing scale/factor (OWS) did evidence concurrent validity with each of the academic performance indicators, the magnitude of the validity coefficients was actually stronger and more consistent for the academic efficacy subscale/factor (AES), which consisted of only one-quarter of the OWS items (Renshaw \& Chenier, 2016).

The generalizability of Renshaw and Chenier's (2016) findings to actual screening practice is questionable, however, given the analytic approach used screener scores as continuous variables and did not transform them into classification models. Given real-life screening in schools requires the use of a grouping or classification mechanism, any study failing to employ such a procedure is not well-matched to practical realities. In order to generate evidence more germane to practice, then, the present study undertook a secondary analysis of Renshaw and Chenier's (2016) dataset-evaluating the relative validity of broad and targeted screening models using analog classifications, which were derived from responses to the OWS and AES. Considering that national or regional norms have yet to be established for 
classifying responses to the SSWQ, it was considered defensible to create local norms from the present sample, as is common practice in school-based screening (Dowdy et al., 2010). We hypothesized that both the OWS and AES screening models would yield evidence in favor of classification validity, and that the relative evidence in favor of classification validity would be stronger for the AES model compared to the OWS model. Taking the term classification validity to refer to meaningful differentiation in criterion performance as a function of screening classification, evidence of this type would suggest that groupings derived from the OWS and AES screening models could be used to effectively discriminate between students with better-and-poorer levels of school performance. For the purposes of this study, stronger evidence in favor of the classification validity of one model compared to the other was operationalized as relatively greater differentiation in students' performance on criterion indicators.

\section{Method}

\section{Participants}

Participants were 335 students enrolled in Grades 5-8 at a public charter school within an urban city in the southeastern United States. The majority of students identified as Black or African American (92.5\%) and qualified for free or reduced-price lunch (94\%). Further detail regarding the sample demographics, context of student participation, data collection, and ethical approval for conducting the research can be found in Renshaw and Chenier (2016).

\section{Measures}

As mentioned above, the SSWQ (Renshaw et al., 2015) is comprised of four, four-item subscales: joy of learning, educational purpose, school connectedness, and academic efficacy. 
These four scales can be scored and interpreted at the subscale level or summed and interpreted at the composite level, making an overall wellbeing scale. Previous research suggests that the SSWQ has a sound higher-order measurement model, and that the four subscales and overall scale are characterized by adequate to strong internal consistency reliability (Renshaw, 2015; Renshaw \& Arslan, 2016). The present study investigated two analog screening models derived from the SSWQ: one based on the Overall Wellbeing Scale (OWS), and the other based only on the Academic Efficacy Subscale (AES). Models based on the other three subscales were not of interest in the present study. Renshaw and Chenier (2016) reported that responses to the SSWQ had good data-model fit with the present sample, and that responses to both the OWS and AES showed strong internal consistency at the group level (OWS $H$-index $=.94$, AES $H$-index $=.81$ ).

Four school-reported academic performance indicators were used to evaluate the classification validity of the OWS and AES screening models: (a) percentile scores from the most recent administration of a nationally-normed mathematics standardized achievement test (referred to hereafter as Math SAT), (b) percentile scores from the companion English language arts standardized achievement test (referred to hereafter as ELA SAT), (c) current grade-point average (GPA), and (d) monthly average "behavioral performance paycheck" scores (referred to hereafter as Paycheck). The Paycheck was a local metric resulting from the school's implementation of a token economy system, which was intended to reinforce students' academic engagement and prosocial behavior (sans academic proficiency or achievement considerations). Paycheck scores were derived for each student by taking the sum of all tokens (delivered by teachers and school staff) earned within the previous month. Higher scores on the 
Paycheck metric can therefore be interpreted as representing recent and relatively greater levels of socially desirable behavior observed by teachers and school staff.

\section{Data Analyses}

Analog screening classifications were created by identifying the 20th percentile score for responses to both the OWS and AES within the present sample to use as cutoff values $\left(20 \%_{\text {ows }}=\right.$ $\left.39,20 \%_{\mathrm{AES}}=10\right)$ and then classifying participants into one of two wellbeing levels based on both the OWS cutoff value (Low $=$ scale score $<39$, Adequate $=$ scale score $\geq 39$ ) and the AES cutoff value $($ Low $=$ scale score $<10$, Adequate $=$ scale score $\geq 10)$. Given national or regional norms are not yet available for the SSWQ, the 20th percentile scores were chosen as the cutoff values based on local-norming logic in school-based service delivery (Shinn, 1988), which is considered reasonable for use within school mental health screening (Dowdy et al., 2010). This procedure resulted in two new categorical variables that represented the broad and targeted screening models. Next, the classification agreement among the OWS and AES screening models was analyzed using a Bayesian contingency table that crossed these categorical variables. Following, the classification validity of both analog screening models was evaluated through a series of Bayesian independent samples $t$-tests that used classification as the grouping variable (Low vs. Adequate) and the four aforementioned academic performance indicators as the dependent variables (Math SAT, ELA SAT, GPA, and Paycheck). All data analyses were conducted using the open-source JASP statistical software (JASP Team, 2018; https://jasp-stats.org).

A Bayesian approach to data analysis was preferred to a more traditional, frequentist approach because resulting evidence could be directly interpreted in light of the hypotheses posed above. Frequentist approaches consider only the extremeness of the data under the null 
hypothesis, and therefore cannot provide direct evidence for the alternative hypothesis.

Findings from frequentist approaches also cannot be used to evaluate the relative evidence in favor of a hypothesis for multiple models or across multiple studies (Wagenmakers, 2007). Bayesian approaches account for these limitations by calculating ratios, called Bayes factors, that contrast the probability of the data fitting under the null hypothesis with the probability of the data fitting under the alternative hypothesis $\left(\mathrm{BF}_{01}\right)$, or vice versa $\left(\mathrm{BF}_{10}\right)$. Bayes factors can therefore be interpreted as evidence to directly address either the null or alternative hypotheses, and can be directly compared across models or studies to evaluate the relative weight of evidence in favor of a given hypothesis (Jarosz \& Wiley, 2014).

In the present study, Bayes factors were calculated using JASP's default prior probability distribution for model parameters. For the $t$-tests, follow-up robustness checks were conducted to evaluate the stability of Bayes factors across a range of Cauchy priors, as recommended by Rouder and colleagues (2009). The relative strength of evidence provided by a given Bayes factor was interpreted using the qualitative guidelines provided within the JASP output: $1-3=$ anecdotal, 3-10 = moderate, 10-30 = strong, and 30+ = very strong (JASP Team, 2018). Additionally, the effect size of posterior distributions was evaluated using the median Glass' $\delta$, which is a standardized mean difference interpreted similarly to Cohen's $d$. Given the nascent state of the wellbeing screening literature and the lack of empirical precedent for doing otherwise, Cohen's (1988) traditional guidelines were adopted for interpreting the magnitude of resulting standardized mean differences: .20-.49 = small, . .50-.79 = medium, .80+ = large. 


\section{Results and Discussion}

Classification contingencies resulting from the 20th percentile cutoffs for both the OWS and AES models are presented in Table 1. The estimated Bayes factor (alternative/null) calculated for these classification contingencies indicated that the data were 45730000000 times more likely to occur under the alternative hypothesis, providing very strong evidence to reject the null hypothesis of no difference between the classifications derived from the broad and targeted models. Furthermore, calculation of the simple agreement between classifications indicated that $46.15 \%$ of those identified as "Low" by the OWS were also identified as "Low" by the AES, and that $62.5 \%$ of those identified as "Low" by the AES were also identified as "Low" by the OWS. Given this evidence, we conclude that the analog screening classifications derived from using the OWS and AES models are not functionally equivalent, as they disagree on a substantial proportion of grouping decisions. Taken at face value, these results suggest that, in practice, the broad and targeted wellbeing screening models are likely to identify large proportions of different students as having mental health risk.

Descriptive statistics for all academic performance indicators are presented by screening model in Table 2. Results from the series of Bayesian independent samples $t$-tests for all academic performance indicators are presented by screening model in Table 3. For the OWS model analyses, resulting Bayes factors (alternative/null) indicated moderate evidence in favor of the alternative hypothesis for Math SAT and very strong evidence in favor of the alternative hypothesis for GPA and Paycheck; however, anecdotal evidence was indicated for the null hypothesis for ELA SAT (see Table 2). For the AES model analyses, resulting Bayes factors (alternative/null) indicated strong evidence in favor of the alternative hypothesis for Math SAT 
and very strong evidence in favor of the alternative hypothesis for ELA SAT, GPA, and Paycheck (see Table 2). Findings from follow-up robustness checks showed that the Bayes factors remained relatively stable across a range of Cauchy priors for the majority of academic performance indicators, providing further support for the strength of evidence in favor of the alternative hypothesis across both screening models. The one exception to this trend was the robustness check for ELA SAT with the AES model, which indicated that evidence in favor of the null hypothesis increased from anecdotal to moderate under wider Cauchy priors. (Note: exact results from these stability analyses are not presented in this brief report, but can be obtained by contacting the authors.)

Considered independently, the Bayes factors resulting from both broad (OWS) and targeted (AES) screening models suggest that students identified as having "Low" wellbeing have substantially poorer school performance across multiple indicators. The median effect sizes $(\delta)$ associated with the posterior distributions for both the OWS and AES screening models further suggests educationally meaningful differences for most academic performance indicators (see Table 3), providing initial evidence in favor of actual between-group differences that are a function of screening classification. When findings from both series of analyses are compared, however, we conclude that the relative evidence in favor of classification validity is stronger for the AES screening model than for the OWS screening model, as the Bayes factors (alternative/null) are greater and the associated median effect sizes are larger across all school performance indicators. Thus, consistent with our hypotheses, the results provide evidence in favor of classification validity for both screening models, yet also show that the evidence for the AES model is stronger than that for the OWS model. 
The practical extension of these analog findings would suggest that actual school-based mental health screening using the AES (targeted) model would identify students with greater levels of school performance risk than would screening using the OWS (broad) model. Considering the additional finding that classifications resulting from both models are functionally non-equivalent, it seems that selecting one model over the other may indeed be a consequential decision for practice. That said, it is noteworthy that the present study is limited by use of a fairly small and demographically heterogenous sample of middle-schoolers, which hinders the generalizability of results to both similar and different populations of students. Prior to recommending these screening models for actual use in practice, then, further research is warranted to replicate and generalize the findings observed herein with both diverse samples of students and across an array of academic performance indicators. Future research may also make use of the analytic approach employed in this study by using Bayes factors to compare the relative weight of evidence in favor of the classification validity of other mental health screening models, such as those based on other approaches (e.g., problems-only vs. problems-plus-wellbeing) or derived from instruments other than the SSWQ. Finally, given all of the remaining unknowns with the student wellbeing screening literature, we suggest researchers and practitioners would do well to select screening models based on a balanced consideration of the best available evidence regarding appropriateness, technical adequacy, and usability within a given context (Glover \& Albers, 2007), not simply classification validity alone. 


\section{References}

Arslan, G., \& Renshaw, T. L. (2018). Student subjective wellbeing as a predictor of adolescents' problem behaviors: A comparison of first-order and second-order factor effects. Child Indicators Research, 11, 507-521. doi:10.1007/s12187-017-9444-0

Cohen, J. (1988). Statistical power analysis for the behavioral sciences (2nd ed.). Hillsdale, NJ: Lawrence Erlbaum.

Cowen, E. L., Dorr, D., Clarifield, S., Kreling, B., McWilliams, S. A., Pokracki, F., Pratt, D. M., Terrell, D., \& Wilson, A. (1973). The AML: A quick-screening device for early identification of school maladaptation. American Journal of Community Psychology, 1, 12-35. doi:10.1007/BF00881243

Dowdy, E., Ritchey, K., \& Kamphaus, R. W. (2010). School-based screening: A population-based approach to inform and monitor children's mental health needs. School Mental Health, 2, 166-176. doi:10.1007/s12310-010-9036-3

Eklund, K., \& Dowdy, E. (2014). Screening for behavioral and emotional risk versus traditional school identification methods. School Mental Health, 6, 40-49. doi:10.1007/s12310-013-9109-1

Furlong, M. J., Gilman, R., \& Huebner, E. S. (2014). Handbook of positive psychology in the schools (2nd ed.). New York, NY: Routledge.

Furlong, M. J., You, S., Renshaw, T. L., Smith, D. C., \& O’Malley, M. D. (2014). Preliminary development and validation of the social and emotional health survey for secondary students. Social Indicators Research, 117, 1011-1032. doi:10.1007/s11205-013-0373-0

Glover, T. A., \& Albers, C. A. (2007). Considerations for evaluating universal screening 
instruments. Journal of School Psychology, 45, 117-135. doi:10.1016/j.jsp.2006.05.005

Jarosz, A. F., \& Wiley, J. (2014). What are the odds? A practical guide to computing and reporting Bayes factors. Journal of Problem Solving, 7, 2-9. doi:10.7771/1932-6246.1167

JASP Team (2018). JASP (Version 0.8.6) [Computer software].

Kim, E. K., Furlong, M. J., Dowdy, E., \& Felix, E. D. (2014). Exploring the relative contributions of the strength and distress components of dual-factor complete mental health screening. Canadian Journal of School Psychology, 29, 127-140. doi:10.1177/0829573514529567

Renshaw, T. L. (2016). Using so-called mind-body practices to promote youths' well-being: Reflections on therapeutic outcomes, strategies, and processes. International Journal of School \& Educational Psychology, 4, 116-121. doi:10.1080/21683603.2016.1130587

Renshaw, T. L. (2015). A replication of the technical adequacy of the Student Subjective Wellbeing Questionnaire. Journal of Psychoeducational Assessment, 33, 757-768. doi:10.1177/0734282915580885

Renshaw, T. L., \& Arslan, G. (2016). Psychometric properties of the Student Subjective Wellbeing Questionnaire with Turkish adolescents: A generalizability study. Canadian Journal of School Psychology, 31, 139-151. doi:10.1177/0829573516634644

Renshaw, T. L., \& Chenier, J. S. (2016). Further validation of the Student Subjective Wellbeing Questionnaire: Comparing first-order and second-order factor effects on actual school outcomes. Journal of Psychoeducational Assessment, 36, 392-397. doi:10.1177/0734282916678494

Renshaw, T. L., Long, A. C. J., \& Cook, C. R. (2015). Assessing adolescents' positive psychological functioning at school: Development and validation of the Student Subjective Wellbeing 
Questionnaire. School Psychology Quarterly, 30, 534-552. doi:10.1037/spq0000088

Rouder, J. N., Speckman, P. L., Sun, D., Morey, R. D., \& Iverson, G. (2009). Bayesian $t$ tests for accepting and rejecting the null hypothesis. Psychonomic Bulletin \& Review, 16, 225-237. doi:10.3758/PBR.16.2.225

Seligman, M. E. P., \& Csikszentmihalyi, M. (2001). Positive psychology: An introduction. American Psychologist, 55, 5-14. doi:10.1037//0003-066X.55.1.5

Shinn, M. R. (1988). Development of curriculum-based local norms for use in special education decision-making. School Psychology Review, 17, 61-80.

Wagenmakers, E-J. (2007). A practical solution to the pervasive problem of $p$ values.

Psychonomic Bulletin \& Review, 14, 779-804. doi:10.3758/BF03194105 
Table 1

Classification Results by Screening Model

\begin{tabular}{llll}
\hline & \multicolumn{2}{l}{ AES } & \\
\cline { 2 - 3 } OWS & Low & Adequate & Total \\
\hline Low & 30 & 35 & 65 \\
Adequate & 18 & 252 & 270 \\
Total & 48 & 387 & 335 \\
\hline
\end{tabular}

Note. OWS = Overall Wellbeing Scale; AES = Academic Efficacy Scale. 
Table 2

Descriptive Statistics of Academic Indicators by Screening Model and Group

\begin{tabular}{llllll}
\hline \multirow{2}{*}{ Model } & \multirow{2}{*}{ Group } & $M(S D)$ & & & \\
\cline { 3 - 6 } & & Math SAT & ELA SAT & GPA & Paycheck \\
\hline \multirow{2}{*}{ OWS } & Low & $30.29(22.03)$ & $32.83(20.55)$ & $2.56(0.59)$ & $49.73(11.76)$ \\
& Adequate & $39.72(25.44)$ & $35.85(24.32)$ & $2.87(0.58)$ & $58.04(13.51)$ \\
\multirow{2}{*}{ AES } & Low & $26.98(21.53)$ & $21.51(17.96)$ & $2.38(0.59)$ & $46.42(11.78)$ \\
& Adequate & $39.55(25.18)$ & $37.46(23.74)$ & $2.88(0.56)$ & $58.08(13.16)$ \\
\hline
\end{tabular}

Note. OWS $=$ Overall Wellbeing Scale; AES = Academic Efficacy Scale. Math SAT $=$ Math standardized achievement test; ELA SAT = English language arts standardized achievement test; GPA = grade-point average. 
Table 3

Results of Bayesian Independent Samples T-Tests by Screening Model

\begin{tabular}{lllll}
\hline \multirow{2}{*}{ Indicator } & \multicolumn{3}{l}{ OWS } & \multicolumn{3}{l}{ AES } \\
\cline { 2 - 5 } & $\mathrm{BF} 10$ & $\delta[95 \% \mathrm{Cl}]$ & $\mathrm{BF} 10$ & $\delta[95 \% \mathrm{Cl}]$ \\
\hline Math SAT & 7.68 & $0.36[0.10,0.64]$ & 21.70 & $0.47[0.15,0.81]$ \\
ELA SAT & 0.37 & $0.15[0.01,0.40]$ & 1127.31 & $0.65[0.34,0.98]$ \\
GPA & 257.35 & $0.52[0.24,0.79]$ & 502809.22 & $0.85[0.54,1.16]$ \\
Paycheck & 2220.49 & $0.60[0.32,0.89]$ & 338779.88 & $0.86[0.54,1.19]$ \\
\hline
\end{tabular}

Note. OWS = Overall Wellbeing Scale; AES = Academic Efficacy Scale. $\mathrm{Cl}=$ credible interval. Math SAT = Math standardized achievement test; ELA = English language arts standardized achievement test; GPA = grade-point average. 